\title{
Predict Lymph Node Metastasis in Penile Cancer Using Clinicopathological Factors and Nomograms
}

\author{
Yanxiang Shao ${ }^{1}, *$ \\ Xiang Tu' ${ }^{l}, *$ \\ Yang Liu',* \\ Yige Bao' \\ Shangqing Ren ${ }^{1,2}$ \\ Zhen Yang ${ }^{1,3}$ \\ Xu Hu (D) ${ }^{1}$ \\ Kan $\mathrm{Wu}^{\prime}$ \\ Hao Zeng' \\ Qiang Wei' \\ Xiang Li (D)
}

'Department of Urology, Institute of Urology, West China Hospital, Sichuan University, Chengdu, People's Republic of China; ${ }^{2}$ Robotic Minimally Invasive Surgery Center, Sichuan Academy of Medical Sciences \& Sichuan Provincial People's Hospital, Chengdu, People's Republic of China; ${ }^{3}$ Department of Urology, Chengdu Second People's Hospital, Chengdu, People's Republic of China

*These authors contributed equally to this work

\begin{abstract}
Objective: To investigate the predictive factors of lymph node metastasis (LNM) and evaluate the usefulness of prediction nomograms.

Methods: This study included 300 patients diagnosed with penile squamous cell carcinoma at West China Hospital (WCH) of Sichuan University (Chengdu, China) and 412 cases acquired from the Surveillance, Epidemiology, and End Results (SEER) program. Logistic regression analysis was performed on these cohorts to investigate the predictive factors of LNM. We evaluated a recently developed prediction nomogram for LNM, which was established based on the National Cancer Database (NCDB). Moreover, we developed a novel nomogram using cases from the WCH for the prediction of lymphatic metastasis.

Results: Logistic analysis identified that younger age at diagnosis, invasion of the penis body, poorer pT stage, $\mathrm{cN}$ stage, nuclear grade and the presence of lymph vascular invasion (LVI) were significantly correlated with LNM in WCH cases; however, only race, poorer $\mathrm{T}$ stage and $\mathrm{cN}$ stage were significantly associated with LNM among the cases from the SEER. Multivariate analysis demonstrated that younger age, poorer $\mathrm{T}$ stage, $\mathrm{cN}$ stage and nuclear grade were independent predictors of LNM. Receiver operating characteristic curve analysis of WCH cases showed that the tumor T stage 8th edition has better area under the curve than 7 th stage $(0.672$ vs 0.636 , respectively). Moreover, well AUC was seen in external validation of NCDB nomogram in WCH cohorts and SEER series $(0.833$ vs 0.795). The new nomogram included the aforementioned independent predictors and the bootstrap-corrected concordance was 0.876 .
\end{abstract}

Conclusion: Younger diagnose age, poorer $\mathrm{pT}$ stage, $\mathrm{cN}$ stage, nuclear grade and LVI were the most important predictors of LNM in patients with penile cancer. 8th T stage performed better than 7th version in predicting LNM. NCDB nomogram has some application values in both WCH and SEER cases, and our novel model further improved the predictive accuracy. Keywords: penile cancer, lymph node metastasis, prediction model, nomogram, SEER

\section{Introduction}

Penile squamous cell carcinoma (PSCC) is a relatively rare genitourinary tumor, with an overall incidence of $<1$ in 100,000 males in the USA and Europe. ${ }^{1,2}$ However, this number is markedly higher and increasing in developing countries. ${ }^{3}$ Metastasis of penile cancer in the inguinal lymph nodes, the most common metastatic site for this type of malignancy, is always associated with a poor prognosis. ${ }^{3,4}$ While inguinal lymph node dissection (ILND) can assist in tumor grading and reduce the risk of mortality, this technique is also associated with an incidence of complications $(70 \%) .{ }^{5,6}$ Hence, it is important to identify patients who will benefit from ILND and to avoid unnecessary surgery.
Correspondence: Xiang $\mathrm{Li}$ Department of Urology, West China Hospital, Sichuan University, 37 GuoXueXiang, Chengdu, Sichuan, People's Republic of China Tel +86 I898060I428

Email xiangli87@hotmail.com 
Previous studies demonstrated that the development of lymph node metastasis (LNM) depends on several clinicopathological factors, such as tumor $\mathrm{T}$ stage, nuclear grade, lymph vascular invasion (LVI), and so on. ${ }^{7,8}$ Models combine these factors could help in the accurate prediction of lymphatic metastasis. ${ }^{9-11}$ Peak et al established a LNM prediction nomogram based on clinicopathological features (nuclear grade, $\mathrm{cN}$ stage and LVI) of patients recorded in the National Cancer Database (NCDB), and this prediction nomogram exhibited high discrimination in its internal validation. ${ }^{11}$ In the present study, we analyzed the predictive value of several clinicopathological factors in patient cohorts from West China Hospital (WCH) of Sichuan University (Chengdu, China) and the Surveillance, Epidemiology, and End Results Program (SEER) database, evaluated the clinical usefulness of the NCDB nomogram, and subsequently developed a novel nomogram using data of PSCC cohort of our institute.

\section{Materials and Methods}

\section{Patient Selection}

This study included patients who were diagnosed with PSCC and underwent complete excision of the lesion through partial or radical penectomy in the Department of Urology at WCH of Sichuan University between September 2008 and October 2020. The exclusion criteria were as follows: 1) presence of unresectable disease or cN3 disease; 2) Eastern Cooperative Oncology Group score $>1$; and 3 ) unwillingness of patients to provide information regarding their disease. Patients provided informed written consent prior to the collection of data. Finally, 300 patients with PSCC were included in this study.

\section{Clinical and Pathological Features}

Patient clinical data (eg, age at diagnosis, smoking history, duration of disease, tumor growth velocity, and clinical lymph node stage) were retrieved from the medical records of our hospital. All pathological reports were provided by the Pathology Department of our institute, including histopathological type, pathological $\mathrm{T}$ stage, tumor size, nuclear grade and LVI. Pathological T stage was adjusted according to the Union for International Cancer Control (UICC) TNM classification system 7th and 8th editions, and the largest diameter of the tumor was recorded to determine its size. Clinical $\mathrm{N}$ stage was recorded at the first outpatient visit, which is 1 month after primary resection.

\section{Follow-Up}

ILND was recommended for patients with pT1G2 or higher stage diseases, and those with palpable inguinal lymph node at the first postoperative outpatient visit. Patients were followed-up through clinical examination once every 3 months during the first year and every 6 months thereafter. Ultrasonography of the groin was performed every 6 months for the first 2 years after surgery. Metastatic inguinal lymph nodes were confirmed by surgical resection or biopsy during follow-up.

\section{SEER Data Resource and Cohort Selection}

All cases of patients with PSCC for whom data were available in the SEER database were examined (SEER Research Data, 18 registries, Nov 2019 Sub; n=5222). We included those who had complete records in terms of $\mathrm{cN}$ stage, tumor nuclear grade, LVI, regional lymph node examined status, survival length and status, and those with cN3 disease were excluded. Except for the abovementioned factors, clinicopathological data (eg, age, race, tumor location, $\mathrm{T}$ stage, tumor size and ILND history) were also collected. Finally, 412 SEER cases were included in our study.

\section{Statistical Methods}

Univariate and multivariate logistic regression analyses were performed to determine the clinicopathological parameters associated with LNM. In univariate analysis, factors with statistical significance were included in the multivariate analysis, and independent predictors of LNM were selected to generate a novel nomogram. Bootstrapping was used to calculate the corrected c-index, and a calibration curve was created. Moreover, patients (WCH and SEER cohorts) were scored using the NCDB nomogram. Receiver operating characteristic curve analysis was used to evaluate predicted value of different clinicopathological factors, the NCDB nomogram and the newly established WCH nomogram. Statistical analyses were performed using the SPSS Statistics 25 (IBM Corporation, Armonk, NY, USA) and the $\mathrm{R}$ Programming Language 4.0.4 (R Foundation for Statistical Computing, Vienna, Austria). A $\mathrm{P}<0.05$ denoted statistically significant differences. 


\section{Results}

Table 1 presents the clinicopathological data of 300 patients with PSCC. The mean age was 54.2 years (standard deviation: 13.9 years), and the median follow-up time was 35.1 months (interquartile range: $15.0-86.6$ months). ILN metastasis occurred in 93 of patients during follow-up. The 2 years cancer-specific-survival rate for lymph node positive or negative series were $53.8 \%$ and $98.9 \%$, respectively. The clinicopathological data of 412 SEER cases were also shown in Table 1. The median follow-up time was 8.5 months (interquartile range: 4.0-15.0 months). In this cohort, LNM occurred in 6 patients during the follow-up. The 2 years cancer-specific-survival rate for lymph node positive and negative groups was $37.5 \%$ and $83.7 \%$ separately.

For the $300 \mathrm{WCH}$ cases, the univariate analysis revealed that younger age at diagnosis, invasion of the penis body, poorer $\mathrm{T}$ stage, $\mathrm{cN}$ stage, nuclear grade and presence of LVI were significantly correlated with ILN metastasis. However, for the 412 SEER cases, only American Indian/Alaska native race, poorer $\mathrm{T}$ stage and cN stage were significantly associated with LNM $(\mathrm{P}<$ 0.05 , Table 2). The multivariate analysis for the $\mathrm{WCH}$ cohort demonstrated that all aforementioned factors, except the location of the lesion, were independent predictors of LNM $(\mathrm{P}<0.05$, Table 3$)$.

Prediction effects of the above independent predictors for LNM were evaluated (Figure 1A and B). In the WCH cases, higher AUCs were seen in $\mathrm{cN}$ stage and nuclear grade, which were 0.754 and 0.722 , respectively; AUCs for all other factors were lower than 0.70, and 8th T stage showed better predictive effect than 7th (AUC: 0.672 vs 0.636 ). In the SEER cases, factors with completed records (grade, LVI and $\mathrm{cN}$ stage) were evaluated, and only cN stage had an AUC higher than 0.70. Besides, external validations of the NCDB nomogram ${ }^{11}$ were performed using both the SEER and WCH cohorts: for these two patient cohorts, the area under the curve (AUC) was 0.833 and 0.795 , respectively (Figure $1 \mathrm{C}$ and $\mathrm{D}$ ).

For better prediction of LNM, a new nomogram was established using clinicopathological data of $\mathrm{WCH}$ cases (Figure 2). All the factors included in the model were previously proven to be independent predictors of LNM. The bootstrap corrected c-index of the model was 0.876, which was similar to the AUC (Figure 1E). Figure 3 illustrates the consistency between the predicted risk and the observed incidence.
Table I Clinicopathological Features for PSCC Patients in $\mathrm{WCH}$ and SEER Database

\begin{tabular}{|c|c|c|}
\hline Variants & $\begin{array}{l}\text { WCH (300 } \\
\text { Patients) }\end{array}$ & $\begin{array}{l}\text { SEER (4 I } 2 \\
\text { Patients) }\end{array}$ \\
\hline \multicolumn{3}{|l|}{ Age of diagnosis (years) } \\
\hline$<50$ & 127 & 36 \\
\hline $50-69$ & 128 & 170 \\
\hline$\geq 70$ & 45 & 206 \\
\hline \multicolumn{3}{|l|}{ Smoking history } \\
\hline No & 141 & NA \\
\hline Yes & 159 & NA \\
\hline \multicolumn{3}{|l|}{ Race } \\
\hline White & 0 & 341 \\
\hline Black & 0 & 44 \\
\hline Asian and Pacific islander & 300 & 15 \\
\hline American Indians and & 0 & 5 \\
\hline Alaska native & & \\
\hline Unknown & 0 & 7 \\
\hline \multicolumn{3}{|l|}{$\begin{array}{l}\text { Tumor growth velocity in } \\
\text { recent } 3 \text { months }\end{array}$} \\
\hline$<\mathrm{Icm} /$ month & 214 & NA \\
\hline$\geq \mathrm{Icm} /$ month & 86 & NA \\
\hline \multicolumn{3}{|l|}{ Tumor location } \\
\hline Prepuse & 15 & 45 \\
\hline Glans penis & 166 & $|5|$ \\
\hline Body of penis & 7 & 24 \\
\hline Overlapping lesion of penis & 112 & 15 \\
\hline Unknown & 0 & 177 \\
\hline \multicolumn{3}{|l|}{ Tumor size } \\
\hline$<\mathrm{Icm}$ & 6 & 39 \\
\hline $\mathrm{I}-4 \mathrm{~cm}$ & 212 & 199 \\
\hline$\geq 4 \mathrm{~cm}$ & 82 & 109 \\
\hline Unknown & 0 & 65 \\
\hline \multicolumn{3}{|l|}{ T stage (7th edition) } \\
\hline $\mathrm{T}_{1}$ & 110 & 224 \\
\hline $\mathrm{T}_{2}$ & $17 \mid$ & 95 \\
\hline $\mathrm{T}_{3}$ & 19 & 6 \\
\hline $\mathrm{T}_{4}$ & 0 & 64 \\
\hline Unknown & 0 & 23 \\
\hline \multicolumn{3}{|l|}{ T stage (8th edition) } \\
\hline$T_{1}$ & 110 & NA \\
\hline $\mathrm{T}_{2}$ & 100 & NA \\
\hline $\mathrm{T}_{3}$ & 90 & NA \\
\hline $\mathrm{T}_{4}$ & 0 & NA \\
\hline \multicolumn{3}{|l|}{ Nuclear grade } \\
\hline Well differentiated & 98 & 123 \\
\hline Moderately differentiated & 146 & 188 \\
\hline Poorly/undifferentiated & 56 & 101 \\
\hline
\end{tabular}

(Continued) 
Table I (Continued).

\begin{tabular}{|l|l|l|}
\hline Variants & $\begin{array}{l}\text { WCH (300 } \\
\text { Patients) }\end{array}$ & $\begin{array}{l}\text { SEER (4 I 2 } \\
\text { Patients) }\end{array}$ \\
\hline $\begin{array}{l}\text { Lymph vascular invasion } \\
\text { Negative or unknown } \\
\text { Positive }\end{array}$ & 278 & $35 \mathrm{I}$ \\
\hline $\begin{array}{l}\text { Clinical N stage } \\
\mathrm{N}_{0}\end{array}$ & 22 & $6 \mathrm{I}$ \\
$\mathrm{N}_{1}$ & 202 & 383 \\
$\mathrm{~N}_{2}$ & 37 & 9 \\
\hline ILND operation & $6 \mathrm{I}$ & 20 \\
No surgery or unknown & 164 & \\
Operation performed & 136 & 397 \\
\hline Positive inguinal lymph node & & 15 \\
No & 207 & 406 \\
Yes & 93 & 6 \\
\hline
\end{tabular}

Abbreviations: $\mathrm{WCH}$, West China Hospital; SEER, Surveillance, Epidemiology, and End Results Program; LVI, lymph vascular invasion; ILND, inguinal lymph node dissection.

\section{Discussion}

The occurrence of PSCC metastasis in the inguinal lymph node is linked to poor prognosis. ${ }^{12}$ Lymph node dissection is the most important approach for the prevention and treatment of LNM. However, surgeons must consider the balance between survival benefit and the high rate of complications. ${ }^{3}$ The use of dynamic sentinel node biopsy has been advocated to avoid unnecessary ILND, though the risk of false-negative results remains inevitable. ${ }^{13}$ Therefore, it is necessary to make an accurate prediction of LNM. This study demonstrated that age at diagnosis, $\mathrm{cN}$ stage and pathological data ( $\mathrm{T}$ stage, nuclear grade and LVI) were independent predictors for LNM. The new nomogram established based on the above factors showed good discrimination. Thus, we consider that it will be helpful in the decision-making regarding ILND.

Previous studies identified several factors associated with LNM of PSCC, such as tumor size, nuclear grade, and LVI, as well as invasion of the corpus, corpus spongiosum, urethra, nerves, etc. ${ }^{7,8,14}$ The UICC pathological T stage combined those factors, which describe the growth and invasion of primary tumors, and was shown to be significantly correlated with LNM. ${ }^{9,10,12}$ The 8th edition of the UICC TNM classification includes the following changes in the definitions of $\mathrm{T} 1, \mathrm{~T} 2$ and $\mathrm{T} 3$ : $\mathrm{T} 1$ was stratified into two different groups depending on LVI; T2 denoted invasion of the corpus spongiosum; and T3 indicated invasion of the corpus cavernosum. ${ }^{15}$ In the present study, cN stage got the highest AUC in both WCH and SEER cohorts, which indicates that postoperative examination of groin area should not be ignored. Moreover, the 8th $\mathrm{T}$ stage showed better AUC than 7 th version, which supports the application of the 8th $\mathrm{T}$ stage when predicting LNM.

This study also demonstrated that younger patients are at a higher risk of developing LNM. Paiva et al retrospectively reviewed 378 patients with PSCC and found that younger patients had a higher frequency of morphological features. ${ }^{16}$ In their study, the frequency of LNM was $49 \% 34 \%$ and $21 \%$ for patients aged $<40$ years, $40-60$ years and $>60$ years, separately. In a study based on SEER database, younger ages were also demonstrated to be independent predictors of LNM, and patients younger than 50 might have more than twice the risk of LNM in comparison with those older than $70 .{ }^{17}$ However, Zhu et al and Peak et al did not report age-related differences with regard to $\mathrm{LNM}^{10,11}$ In fact, onset age is a factor that has been neglected for a long time: its predictive value for the development of LNM and prognosis should be further validated. Additionally, further investigations on the clinicopathological characteristics of younger patients with PSCC are warranted.

A nomogram based on the aforementioned prediction factors has been established to accurately predict the occurrence of LNM. In 2006, Ficarra et al, reported a nomogram that predicted LNM in patients with PSCC. ${ }^{9}$ A total of 175 patients were included in their study, and the nomogram was produced using the following factors: tumor thickness, growth pattern, grade, embolization, invasion of the corpus cavernosum, corpus spongiosum and urethra, and $\mathrm{cN}$ stage. In their internal validation, the AUC of the nomogram was 0.876 . Over the years, researchers have also tried to improve the accuracy of predictions by including more lab and pathology findings. In 2010, Zhu et al developed a nomogram, which included 110 patients and combined $\mathrm{T}$ stage, grade, LVI and p53 expression. ${ }^{10}$ The internal validation showed a c-index of 0.79 . An external validation, performed by Maciel et al, 65 patients yield a c-index of $0.783 .{ }^{18} \mathrm{Hu}$ et al established a LNM predictive nomogram, which combined programmed death ligand 1 expression level, the neutrophil-to-lymphocyte ratio, LVI and tumor grade. ${ }^{19}$ In their internal validation of 84 patients, c-index was 0.89. ${ }^{19}$ However, these laboratory and pathological 
Table 2 Univariate Analysis of PSCC Patients in WCH and SEER Database

\begin{tabular}{|c|c|c|c|c|c|c|}
\hline \multirow[t]{2}{*}{ Clinical Pathological Data } & \multicolumn{3}{|c|}{ WCH (300 Patients) } & \multicolumn{3}{|c|}{ SEER (4 I 2 Patients) } \\
\hline & $P$ value & Risk Ratio & $95 \% \mathrm{Cl}$ & $P$ value & Risk Ratio & $95 \% \mathrm{Cl}$ \\
\hline Smoking $^{\mathrm{a}}$ & 0.872 & 1.041 & $0.639-1.695$ & NA & NA & NA \\
\hline $50 \leq$ Diagnosis age $<70$ years $^{\mathrm{b}}$ & 0.058 & 0.603 & $0.357-1.018$ & 0.998 & NA & NA \\
\hline Diagnosis age $>70$ years $^{\mathrm{b}}$ & 0.021 & 0.385 & $0.17 I-0.868$ & 0.998 & NA & NA \\
\hline Black $^{c}$ & NA & NA & NA & 0.552 & 1.959 & $0.214-17.935$ \\
\hline Asian or Pacific Islander ${ }^{c}$ & NA & NA & NA & 0.999 & NA & NA \\
\hline American Indian/Alaska Native ${ }^{c}$ & NA & NA & NA & 0.013 & 21.063 & $1.905-232.833$ \\
\hline Glans penis $^{d}$ & 0.892 & 1.086 & $0.329-3.58 \mid$ & 1.000 & NA & NA \\
\hline Body of penis ${ }^{d}$ & 0.022 & 16.500 & $1.487-183.070$ & 0.998 & NA & NA \\
\hline Overlapping lesion of penis ${ }^{d}$ & 0.576 & 1.412 & $0.421-4.733$ & 0.998 & NA & NA \\
\hline $\mathrm{Icm} \leq$ Tumor size $<4 \mathrm{~cm}^{\mathrm{e}}$ & 0.414 & 2.465 & $0.283-21.502$ & 0.998 & NA & NA \\
\hline Tumor size $\geq 4 \mathrm{~cm}^{\mathrm{e}}$ & 0.517 & 2.069 & $0.229-18.656$ & 0.998 & NA & NA \\
\hline Tumor growth $\geq \mathrm{Icm} /$ month $^{f}$ & 0.540 & 0.843 & $0.488-1.456$ & NA & NA & NA \\
\hline 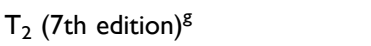 & 0.001 & 4.376 & $2.377-8.059$ & 0.388 & 2.387 & $0.331-17.200$ \\
\hline $\mathrm{T}_{3}(7 \text { th edition })^{g}$ & 0.076 & 2.712 & $0.900-8.171$ & 0.018 & 22.200 & $1.718-286.849$ \\
\hline $\mathrm{T}_{4}{\text { (7th edition })^{g}}$ & NA & NA & NA & 0.997 & NA & NA \\
\hline $\mathrm{T}_{2}$ (8th edition) ${ }^{\mathrm{g}}$ & 0.001 & 3.305 & $1.693-6.452$ & NA & NA & NA \\
\hline $\mathrm{T}_{3}$ (8th edition) ${ }^{\mathrm{g}}$ & 0.001 & 5.375 & $2.744-10.529$ & NA & NA & NA \\
\hline $\mathrm{T}_{4}$ (8th edition) $)^{g}$ & NA & NA & NA & NA & NA & NA \\
\hline Moderately differentiated ${ }^{\mathrm{h}}$ & 0.001 & 5.470 & $2.546-11.752$ & 0.557 & 1.978 & $0.203-19.239$ \\
\hline Poorly/undifferentiated ${ }^{h}$ & 0.001 & 15.283 & $6.400-36.493$ & 0.464 & 2.465 & $0.220-27.580$ \\
\hline LVI present/identified' & 0.001 & 6.717 & $2.537-17.787$ & 0.997 & NA & NA \\
\hline $\mathrm{cN}_{1}{ }^{\mathrm{i}}$ & 0.005 & 3.008 & I.407-6.430 & 0.001 & 54.429 & $6.68 I-443.449$ \\
\hline $\mathrm{CN}_{2}{ }^{\mathrm{i}}$ & 0.001 & 16.588 & $8.226-33.452$ & 0.003 & 21.167 & $2.819-158.952$ \\
\hline
\end{tabular}

Notes: ${ }^{a}$ Reference group is no-smoker; ${ }^{b}$ reference group is diagnosis age $<50$; ${ }^{c}$ reference group is White patients; ${ }^{d}$ reference group is those tumor in prepuse; ${ }^{\mathrm{e}}$ reference group is tumor

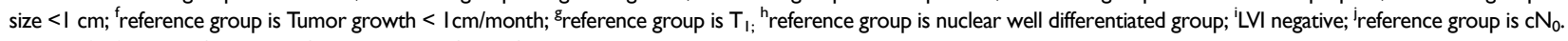
Abbreviations: WCH, West China Hospital; SEER, Surveillance, Epidemiology, and End Results Program; LVI, lymph vascular invasion.

Table 3 Multivariate Analysis of PSCC Patients in WCH Cases

\begin{tabular}{|c|c|c|c|}
\hline \multirow[t]{2}{*}{ Clinical Pathological Data } & \multicolumn{3}{|c|}{ WCH 300 Patients } \\
\hline & $P$ value & Risk Ratio & $95 \% \mathrm{Cl}$ \\
\hline $50 \leq$ Diagnosis age $<70$ years $^{\mathrm{a}}$ & 0.063 & 0.512 & $0.252-1.038$ \\
\hline Diagnosis age $>70$ years $^{\mathrm{a}}$ & 0.004 & 0.199 & $0.066-0.602$ \\
\hline Glans penis ${ }^{\mathrm{b}}$ & 0.218 & 0.397 & $0.091-1.725$ \\
\hline Body of penis ${ }^{\mathrm{b}}$ & 0.467 & 3.105 & $0.147-65.743$ \\
\hline Overlapping lesion of penis ${ }^{b}$ & 0.103 & 0.275 & $0.058-1.300$ \\
\hline$T_{2}$ (8th edition) ${ }^{c}$ & 0.052 & 2.531 & $0.991-6.469$ \\
\hline $\mathrm{T}_{3}$ (8th edition) ${ }^{\mathrm{c}}$ & 0.005 & 3.975 & $1.527-10.350$ \\
\hline Moderately differentiated $^{d}$ & 0.005 & 3.954 & $1.526-10.248$ \\
\hline Poorly/undifferentiated ${ }^{d}$ & 0.001 & $|4.86|$ & $4.930-44.795$ \\
\hline LVI present/identified ${ }^{\mathrm{e}}$ & 0.054 & 3.463 & $0.978-12.259$ \\
\hline $\mathrm{cN}_{\mathrm{l}}{ }^{\mathrm{f}}$ & 0.028 & 2.679 & $1.114-6.442$ \\
\hline $\mathrm{CN}_{2}{ }^{\mathrm{f}}$ & 0.001 & 14.642 & $6.303-34.013$ \\
\hline
\end{tabular}

Notes: ${ }^{a}$ Reference group is diagnosis age $<50$; ${ }^{b}$ reference group is tumor in prepuse; ${ }^{C}$ Reference group is $T_{1 ;}{ }^{d}$ reference group is nuclear well differentiated group; ${ }^{e} \mathrm{LVI}$ negative; ${ }^{\mathrm{f}}$ reference group is $\mathrm{CN}_{0}$.

Abbreviations: WCH, West China Hospital; LVI, lymph vascular invasion. 
A ROC curve of different variants for WCH cases

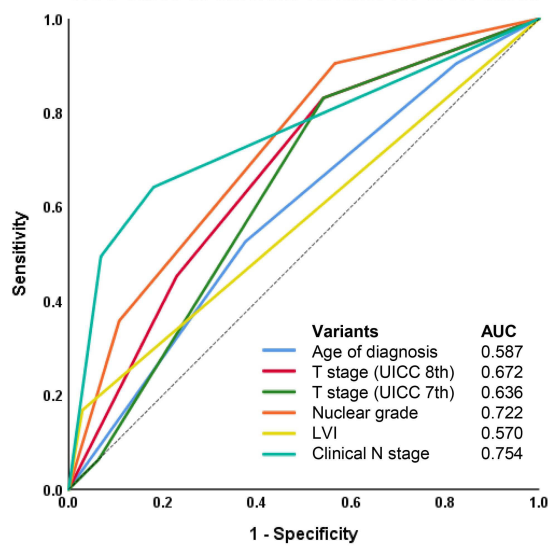

C ROC curve of NCDB nomogram for WCH cases

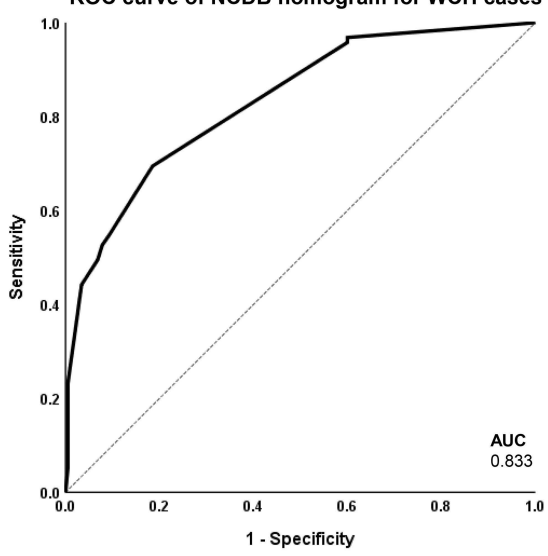

B ROC curve of different variants for SEER cases

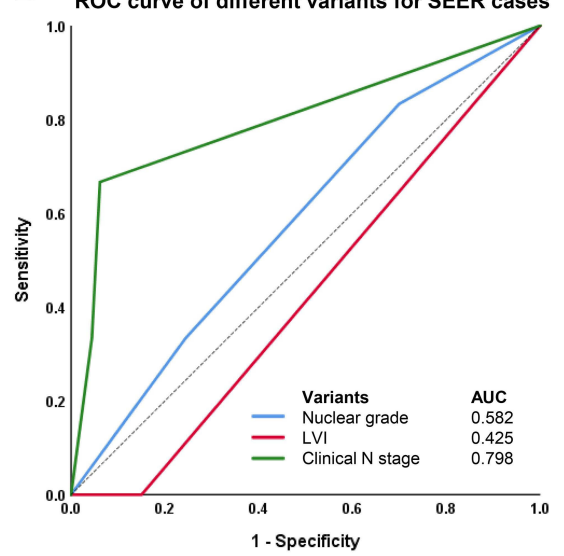

D ROC curve of NCDB nomogram for SEER cases

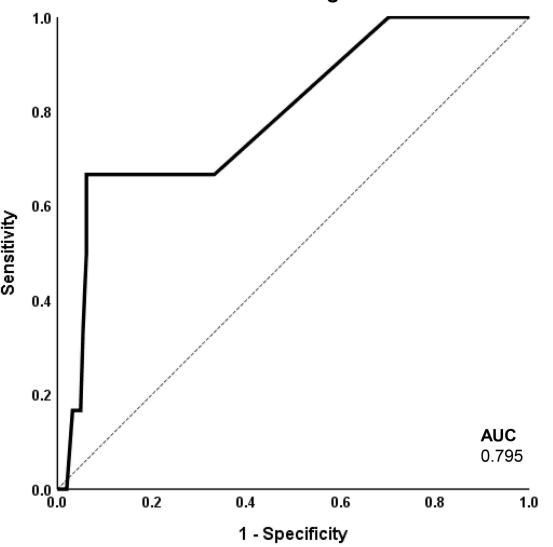

E ROC curve of new nomograms for WCH cases

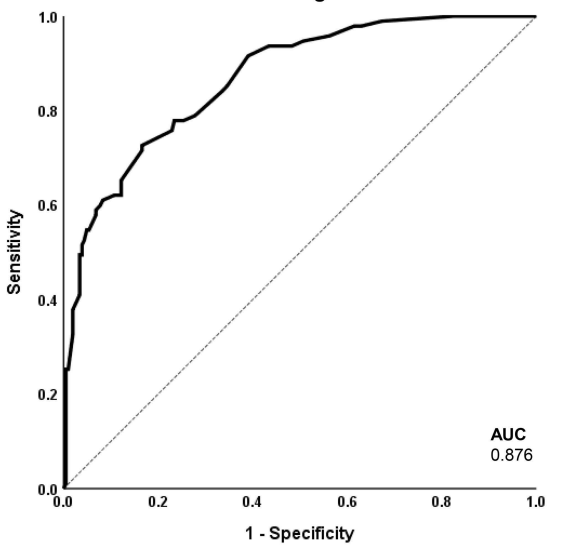

Figure I ROC curve of different variants and nomograms predicting LNM. (A) ROC curve of different variants for WCH cases. (B) ROC curve of different variants for SEER cases. (C) ROC curve of NCDB nomogram for WCH cases. (D) ROC curve of NCDB nomogram for SEER cases; (E) ROC curve of new nomograms for WCH cases. Abbreviations: ROC, receiver operating characteristic curve; LNM, lymph node metastasis; AUC, area under the curve; WCH, West China Hospital; SEER, Surveillance, Epidemiology, and End Results Program; UICC, Union for International Cancer Control; LVI, lymph vascular invasion.

indicators are not routinely tested in most institutions. In recent years, a more simplified nomogram was established based on 1636 patients from the NCDB. ${ }^{11}$ This model included grade, $\mathrm{cN}$ stage and LVI, and its internal validation produced an AUC of 0.880. Our present study showed relatively high accuracy of external verification in both $\mathrm{WCH}$ and SEER cases, which reflected the role of grade, $\mathrm{cN}$ and LVI in the prediction of LNM. After combining these factors with the latest $\mathrm{T}$ stage and diagnostic age, we obtained a high c-index of 0.876 . It is anticipated that this novel model may have greater application value for Asian population to which our patient series belongs to.

There were some limitations in this study. First, the population included in our nomogram is derived from a single source. Although some systematic errors (such as religion, race and medical-care conditions) could be reduced, the usefulness of this model in other geographic regions and populations of other racial backgrounds could not be evaluated. Furthermore, we did not analyze molecular targets that may be associated with LNM. The present model only incorporates the most important and routine clinicopathological factors; thus, we hope that this approach may facilitate to clinical practice and its further evaluation.

\section{Conclusions}

For patients with PSCC, age at diagnosis, pathological $\mathrm{T}$ stage, nuclear grade, LVI, and $\mathrm{cN}$ stage were independent predictors of LNM. The UICC 8th T stage has better predictive value for LNM than 7th edition. The NCDB nomogram has acceptable predictive value in $\mathrm{WCH}$ and SEER series. In this study, a novel prediction nomogram for LNM was generated based on WCH cases. This model incorporates the aforementioned independent-prediction factors and shows good predictive power. 
Points

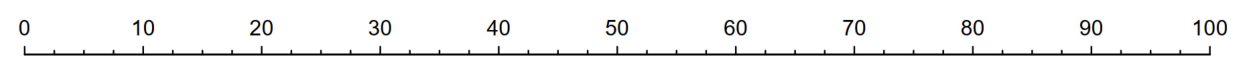

Age at diagnosis

$\geq 70$ years $\stackrel{50-69 \text { years }}{2}<50$ years

Pathological T stage

pT2

pT1 pT3

Nuclear grade

Moderately differentiated
${ }$ Poorly/ undifferentiated $}$

LVI

Negative Positive

Clinical N stage

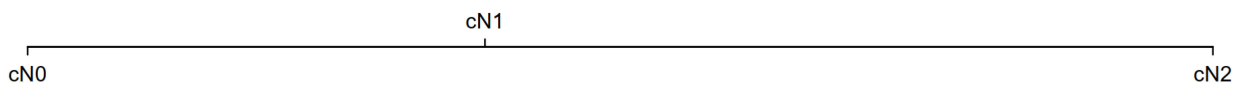

Total points

\begin{tabular}{lllllllll}
\hline 0 & 50 & 100 & 150 & 200 & 250 & 300 & 350 & 400
\end{tabular}

LNM rate

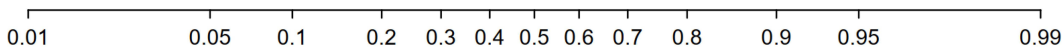

Figure 2 Novel nomogram predicting LNM in patients with $\mathrm{cN}_{0-2}$ PSCC.

Abbreviations: PSCC, penile squamous cell carcinoma; LVI, lymph vascular invasion; LNM, lymph node metastasis.

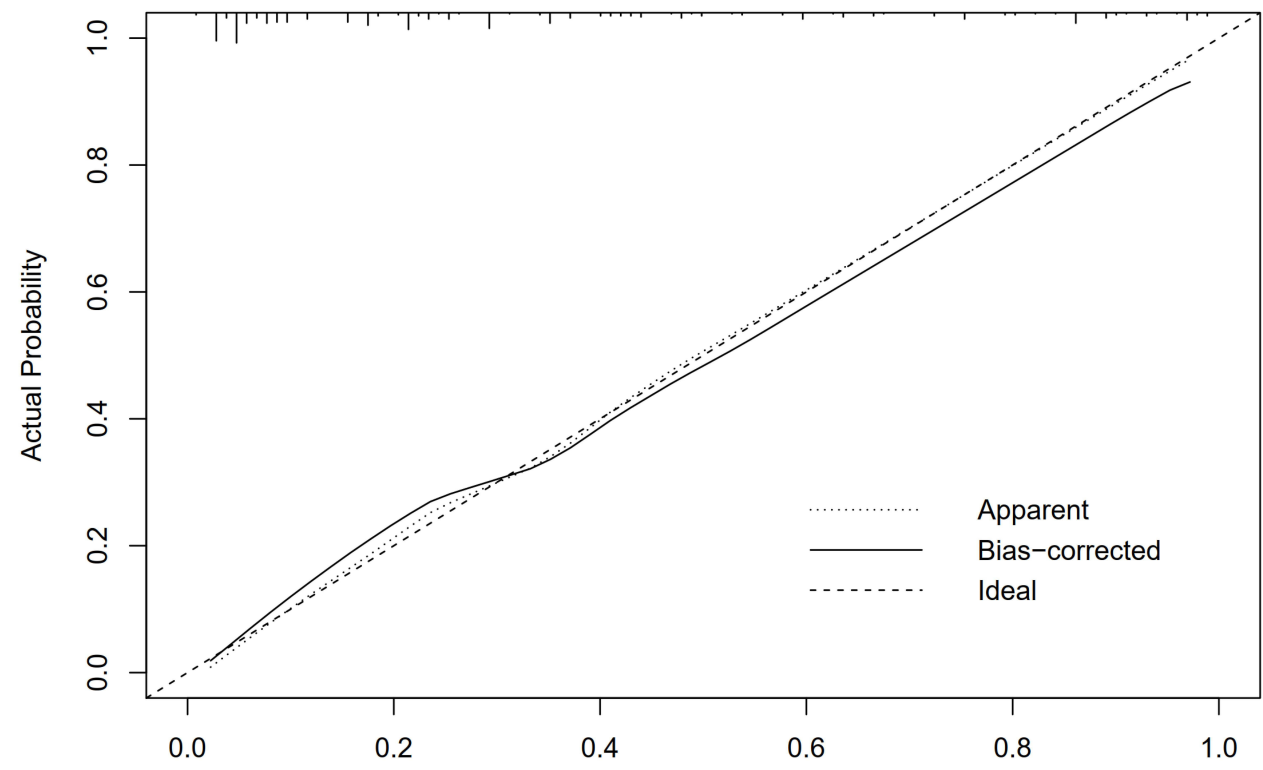

Nomogram Predicted Probability of Positive Nodes

Figure 3 Calibration plot for $\mathrm{WCH}$ novel nomogram predicting LNM. 


\section{Data Sharing Statement}

The data that support the findings of this study are available on request from the corresponding author.

\section{Ethics Approval and Consent to Participate}

This retrospective study was approved by the Ethics Committee of West China Hospital, Sichuan University, with the whole process supervised. Patients and their authorized family members had been fully informed before follow-up work was performed, with informed consent signed. All this study was conducted in accordance with the Declaration of Helsinki.

\section{Consent for Publication}

Consent for publication was obtained from all participants.

\section{Acknowledgments}

We would like to thank the Surveillance, Epidemiology, and End Results database for providing high-quality clinical data for our research.

\section{Funding}

This study was supported by the National Natural Science Foundation of China (Reference Number: 81672552), the Science and Technology Foundation of Sichuan Province (2017JY0226) and the 1.3.5 Project for Disciplines of Excellence, West China Hospital, Sichuan University (Reference number: ZY2016104).

\section{Disclosure}

The authors report no conflicts of interest in this work.

\section{References}

1. Backes DM, Kurman RJ, Pimenta JM, Smith JS. Systematic review of human papillomavirus prevalence in invasive penile cancer. Cancer Causes Control. 2009;20(4):449-457. doi:10.1007/s10552008-9276-9

2. Chaux A, Netto GJ, Rodriguez IM, et al. Epidemiologic profile, sexual history, pathologic features, and human papillomavirus status of 103 patients with penile carcinoma. World J Urol. 2013;31(4):861-867. doi:10.1007/s00345-011-0802-0

3. Pettaway CA, Crook JM, Pagliaro LC. Tumors of the Penis. 11th ed. Wein AJ, editor. Philadelphia: Elsevier; 2016.

4. Wen S, Ren W, Xue B, et al. Prognostic factors in patients with penile cancer after surgical management. World J Urol. 2018;36(3): 435-440. doi:10.1007/s00345-017-2167-5
5. Koifman L, Hampl D, Koifman N, Vides AJ, Ornellas AA. Radical open inguinal lymphadenectomy for penile carcinoma: surgical technique, early complications and late outcomes. J Urol. 2013;190 (6):2086-2092. doi:10.1016/j.juro.2013.06.016

6. Delman KA, Kooby DA, Rizzo M, Ogan K, Master V. Initial experience with videoscopic inguinal lymphadenectomy. Ann Surg Oncol. 2011;18(4):977-982. doi:10.1245/s10434-010-1490-5

7. Slaton JW, Morgenstern N, Levy DA, et al. Tumor stage, vascular invasion and the percentage of poorly differentiated cancer: independent prognosticators for inguinal lymph node metastasis in penile squamous cancer. $J$ Urol. 2001;165(4):1138-1142. doi:10.1016/ S0022-5347(05)66450-6

8. Graafland NM, Lam W, Leijte JA, et al. Prognostic factors for occult inguinal lymph node involvement in penile carcinoma and assessment of the high-risk EAU subgroup: a two-institution analysis of 342 clinically node-negative patients. Eur Urol. 2010;58(5):742-747. doi:10.1016/j.eururo.2010.08.015

9. Ficarra V, Zattoni F, Artibani W, et al. Nomogram predictive of pathological inguinal lymph node involvement in patients with squamous cell carcinoma of the penis. J Urol. 2006;175(5):1700-4; discussion 4-5. doi:10.1016/S0022-5347(05)01003-7

10. Zhu Y, Zhang HL, Yao XD, et al. Development and evaluation of a nomogram to predict inguinal lymph node metastasis in patients with penile cancer and clinically negative lymph nodes. $J$ Urol. 2010;184(2):539-545. doi:10.1016/j.juro.2010.03.145

11. Peak TC, Russell GB, Dutta R, Rothberg MB, Chapple AG, Hemal AK. A national cancer database-based nomogram to predict lymph node metastasis in penile cancer. BJU Int. 2019;123 (6):1005-1010. doi:10.1111/bju.14652

12. Alkatout I, Naumann CM, Hedderich J, et al. Squamous cell carcinoma of the penis: predicting nodal metastases by histologic grade, pattern of invasion and clinical examination. Urol Oncol. 2011;29 (6):774-781. doi:10.1016/j.urolonc.2009.10.014

13. Lam W, Alnajjar HM, La-Touche S, et al. Dynamic sentinel lymph node biopsy in patients with invasive squamous cell carcinoma of the penis: a prospective study of the long-term outcome of 500 inguinal basins assessed at a single institution. Eur Urol. 2013;63(4):657-663. doi:10.1016/j.eururo.2012.10.035

14. Hu J, Cui Y, Liu P, et al. Predictors of inguinal lymph node metastasis in penile cancer patients: a meta-analysis of retrospective studies. Cancer Manag Res. 2019;11:6425-6441. doi:10.2147/CMAR.S20 6579

15. Brierley J. TNM Classification of Malignant Tumours. 8th ed. International Union Against Cancer, Wiley-Blackwell; 2016:18 8-189.

16. Paiva GR, de Oliveira Araujo IB, Athanazio DA, de Freitas LA. Penile cancer: impact of age at diagnosis on morphology and prognosis. Int Urol Nephrol. 2015;47(2):295-299. doi:10.1007/ s11255-014-0875-y

17. Zhang W, Gao P, Gao J, Wu X, Liu G, Zhang X. A clinical nomogram for predicting lymph node metastasis in penile cancer: a SEER-Based Study. Front Oncol. 2021;11:640036. doi:10.3389/ fonc. 2021.640036

18. Maciel CVM, Machado RD, Morini MA, et al. External validation of nomogram to predict inguinal lymph node metastasis in patients with penile cancer and clinically negative lymph nodes. Int Braz J Urol. 2019;45(4):671-678. doi:10.1590/s1677-5538.ibju.2018.0756

19. $\mathrm{Hu} \mathrm{J}, \mathrm{Li} \mathrm{H}, \mathrm{He}$ T, et al. A nomogram incorporating PD-L1, NLR, and clinicopathologic features to predict inguinal lymph node metastasis in penile squamous cell carcinoma. Urol Oncol. 2020;38(7):641e19641e29. doi:10.1016/j.urolonc.2020.04.015 


\section{Publish your work in this journal}

Cancer Management and Research is an international, peer-reviewed open access journal focusing on cancer research and the optimal use of preventative and integrated treatment interventions to achieve improved outcomes, enhanced survival and quality of life for the cancer patient.

The manuscript management system is completely online and includes a very quick and fair peer-review system, which is all easy to use. Visit http://www.dovepress.com/testimonials.php to read real quotes from published authors. 